\title{
Prediction Model for Disease Progression and Relevant Prognostic Factors in Hodgkin Patients: An Application of Frailty Multistate Model
}

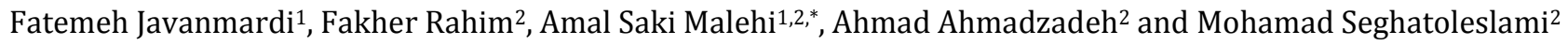 \\ ${ }^{1}$ Department of Biostatistics and Epidemiology, Faculty of Health, Ahvaz Jundishapur University of Medical Sciences, Ahvaz, Iran \\ 2 Research Center of Thalassemia and Hemoglobinopathy, Health Research Institute, Ahvaz Jundishapur University of Medical Sciences, Ahvaz, Iran
}

* Corresponding author: Amal Saki Malehi, Department of Biostatistics and Epidemiology, Faculty of Health, Ahvaz Jundishapur University of Medical Sciences, Ahvaz, Iran; Research Center of Thalassemia and Hemoglobinopathy, Health Research Institute, Ahvaz Jundishapur University of Medical Sciences, Ahvaz, Iran. Tel: 06133115311; Email: amalsaki@gmail.com

Received 2020 January 16; Revised 2021 February 01; Accepted 2021 February 23.

\begin{abstract}
Background: In clinical cancer studies, there has been a high tendency of searching for more specific and new prognostic factors in cancers in the last few years.

Objectives: This multistate study aimed to model the progression of Hodgkin's disease by accounting for individual effect (heterogeneity) using the joint and independent frailty models.

Methods: After the utilization of the illness-death model, joint-modeling accounted for the dependency between relapse and death by considering the individual characteristics as a frailty term. Therefore, the effect of influential prognostic factors was evaluated on disease progression by frailty and joint-frailty multistate models.

Results: The individual predictions were determined using the frameworks of the both models. The model was applied to 389 Hodgkin lymphoma patients. Gender (male), age (over 55 years), and low level of hemoglobin (less than 10.5) were associated with an increased risk of death and relapse in patients. The likelihood cross-validation criterion was proposed to choose the joint frailty model as a better fitting model.

Conclusion: Multistate models were appropriate tools to study the whole event history of the subjects, which provided a deep insight into the dynamics of the disease. The problem of events-subjects dependency in the survival data was clarified using the multistate model. Therefore, the heterogeneity and dependency between the states led to more accurate estimations of the effects of the prognos tic factors, thereby improving the predictions.
\end{abstract}

Keywords: Disease progression, Hodgkin lymphoma, Joint frailty, Multistate model, Prediction

\section{Background}

Lymphoma is a common name for cancers that affect the lymphatic system. Moreover, it is known as the cancer of white blood cells and is categorized into two subtypes of Hodgkin and non-Hodgkin. One of the high achievements in cancers is the progress in the Hodgkin Lymphoma (HL) treatment during the last years. Despite recent progress, a small proportion of patients experience a relapse or disease refractory during initial treatment (1). Although treatment of the advanced stage of the disease has improved, $10 \%$ of patients fail to reach complete remission, and $20 \%-30 \%$ of responding patients experience relapse post-treatment (2). The individual characteristic or patient's frailty has impacts on the overall survival of patients after diagnosing the disease and their response to treatment method (3). Relapse or distant metastases is an important subject that may affect a large number of cancer patients, causing death. The exact cause of the disease has not been clear yet. However, many clinical and laboratory factors are associated with an increased risk of recurrence (4). The first challenge raised by clinical cancer research is to evaluate the prognostic factors. In fact, an important issue is to determine how these factors can affect the disease progression and survival of the patients.

After the diagnosis and the treatment of the primary tumor, local relapses or distant metastases can appear sometimes either after a short time or after years. In some diseases, such as cancer, relapse may affect the instantaneous risk of death (5). Therefore, it is also important for the patient to receive the most appropriate treatment after each relapse. The use of a common statistical approach, which evaluates the occurring time of an event, is unable to indicate movements of patients between different states.

Multistate Models (MSMs) allow an evaluation of the effect of different treatments and prognostic factors on the natural progress of a disease. Frailty and joint frailty MSMs are two main survival models that consider the problem of heterogeneity. This is due to individual effects that are known as unobserved random effects (6). They provide a framework to jointly model the hazards of various types of events, where the occurrence of each event can affect the risk of the other event. Despite a number ofseveral types of research on Hodgkin's disease, a lack of a novel survival statistical analysis is observed in the processing of the disease. Therefore, 
the MSM is useful in managing the transition period in the disease while detecting the effect of other baseline and clinical characteristics on survival.

\section{Objectives}

This study aimed to model the progression of Hodgkin disease by studying the individual effect (heterogeneity) using the joint and independent frailty models. The effect of influential prognostic factors was also evaluated in disease progression.

\section{Methods}

\subsection{Study design}

This retrospective cohort study was performed on 389 patients with HL, who were treated at hematology and oncology clinic, Shafa Hospital, Ahvaz, southwestern Iran, during 2002-2012. The study protocol was approved by the Research Ethics Committee of Ahvaz Jundishapur University of Medical Sciences, Ahvaz, Iran (IR.AJUMS.REC.1396.6). It should be mentioned that informed consent was obtained from all patients. Furthermore, the patient's disease was confirmed by histology based on the decision of two pathologists. In this study, demographic and laboratory data, the final status of patients in terms of death or recurrence, stage, treatment, and the histologic type were collected using a standardized data extraction form.

\subsection{Participants}

This study included patients who were over 15 years of age. On the other hand, the patients with incomplete information were excluded from the study. The follow-up duration for all the cases ranged from 2 to 15 years. Furthermore, the cases were staged clinically according to the Ann Arbor staging system. The entire transition hazard, as a dependent variable, was estimated for age, gender, lymph location, biopsy, aspiration (removal by the suction of fluid and cells through a needle), hemoglobin, and classical Hodgkin. Since the stages of the disease were missing in most of the documents, no appropriate model was fitted in this study.

\subsection{Statistical analysis}

An MSM was used to explain the time of disease diagnosis until death considering an intermediate event. In fact, transition intensities were assessed between a set of defined states using the MSM. However, Cox regression models were used for every endpoint separately and did not illustrate what happened to patients after each event. The multistate model used in this study was the illness-death model with three states, one of which was an absorbing state (death). The illness-death model was implemented by considering the independent frailty and joint frailty between relapse and death. Models with frailty inclusion accounted for unobserved risk factors that were common to all subjects belonging to groups, such as hospitals, families, and countries. The represented frailty was considered the effect of personality characteristics for all members. Even though various frailty distributions existed, commonly, the frailty follows Gamma distribution. The transition intensity by the inclusion of frailty is defined as below (6):

$$
\lambda_{i j}^{D}\left(t \mid u_{i}\right)=\lambda_{0}{ }^{D}(t) \exp \left(\beta_{1}^{\prime} Z_{i j}{ }^{D}\right)
$$

Intensity function with a joint frailty term is as follows (6):

$$
\left\{\begin{array}{l}
\lambda_{i j}{ }^{R}\left(t \mid u_{i}\right)=u_{i} \lambda_{0}{ }^{R}(t) \exp \left(\beta_{1}^{\prime} Z_{i j}{ }^{R}\right)=u_{i} \lambda_{i j}{ }^{R}(t) \\
\lambda_{i}{ }^{D}\left(t \mid u_{i}\right)=u^{\alpha}{ }_{i} \lambda_{0}{ }^{D}(t) \exp \left(\beta_{2}{ }^{\prime} Z_{i}{ }^{D}\right)=u_{i}{ }^{\alpha} \lambda_{i}{ }^{D}(t)
\end{array}\right\}
$$

In fact, in the joint frailty multistate model, different times to transitions were dependent. In this study, relapse and death hazards (terminal event) were jointly modeled $\left(\alpha_{01} \rightarrow \alpha_{12}\right)$. The multistate diagram (Figure 1) shows the clinical state that a patient could occupy at any time during the followup. The arrows denoted in Figure 1 indicated the direction of possible transitions. The statistical inference about the joint model proposed by Rondeau was based on the semi-parametric penalized likelihood approach that was the most suitable selected model using the likelihood of crossvalidation criteria (7).

Parameter vectors for relapse (R) and death (D) were denoted by $\xi=\left(\lambda_{0}{ }^{R}(),. \lambda_{0}{ }^{D}(),. \beta, \alpha, \theta\right)$. Moreover, the following expression shows the full log-likelihood for subject $\mathrm{i}(\mathrm{i}=1, \ldots \mathrm{N})$, where $\delta_{i j}{ }^{R}$ is an indicator, which is 0 if patients died and 1 if $j$ th relapse is observed (7):

The baseline hazard function $\left(\lambda_{0}{ }^{R}(\right.$.$\left.) and \lambda_{0}{ }^{D}().\right)$ was estimated by Splines. Therefore, the penalized

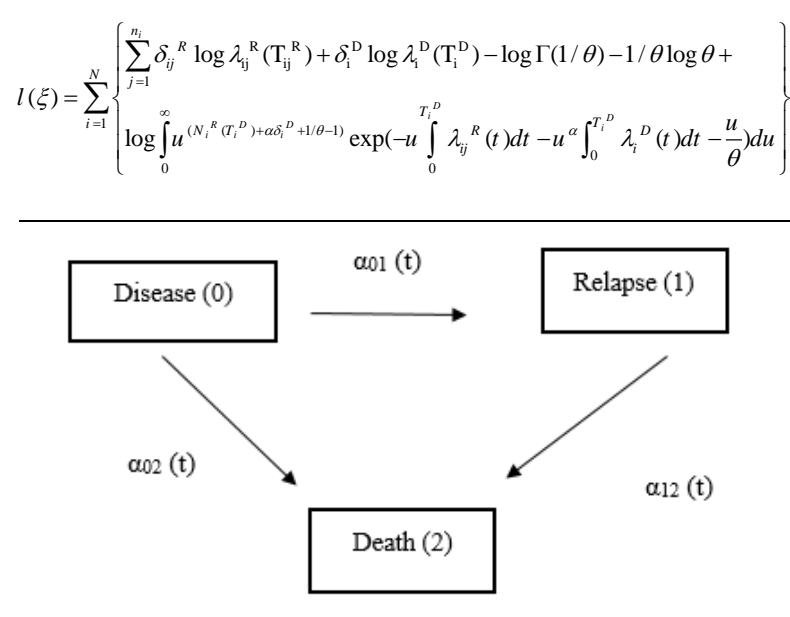

Figure 1. Illness-death model 
log-likelihood is as follows:

$$
\operatorname{lpl}(\xi)=l(\xi)-k_{1} \int_{0}^{\infty}\left\{\left(\lambda_{0}{ }^{R}\right)^{\prime \prime}(t)\right\}^{2} d t-k_{2} \int_{0}^{\infty}\left\{\left(\lambda_{0}{ }^{D}\right)^{\prime \prime}(\mathrm{t})\right\}^{2} d t
$$

Where, $k_{1}$ and $k_{2}$ are two positive smoothing parameters. All the calculations were performed by an existing R package and Frailty Pack for both the models and predictions (7).

\section{Results}

This study investigated 389 patients, who had been diagnosed with HL. It should be mentioned that the majority $(n=228 ; 58.6 \%)$ of the cases were male. Regarding the age group, 52 (13.36\%) cases were less than 15 years of age, and 241 (61.95\%) patients were between 15 and 34 years. Moreover, 70 (18\%) and $26(6.69 \%)$ individuals were within the range of 34-55 and over 55 years, respectively. The mean \pm SD age at diagnosis was $27.5 \pm 15.83$ years, and the median follow-up was estimated at 5.66 years. The transitional information of the patients is presented in Table 1 . As can be observed, 30 and 23 patients died following relapse and without a previous relapse, respectively.

According to Ann Arbor staging system, the cases were classified as grades I ( $\mathrm{n}=82 ; 21.1 \%)$, II $(\mathrm{n}=85$; $21.9 \%)$, III ( $n=96 ; 24.7 \%)$, and IV ( $n=24 ; 6.20 \%)$.

\begin{tabular}{lccc}
\hline \multicolumn{4}{l}{ Table 1. Summary of the number of transitions for each state } \\
\hline State & 1 (Disease) & 2 (Relapse) & 3 (Death) \\
\hline 1 (Disease) & $226(58.09 \%)$ & $136(34.96 \%)$ & $23(5.91)$ \\
2 (Relapse) & - & $99(25.44)$ & $30(7.71)$ \\
\hline
\end{tabular}

However, other patients had an incomplete medical history. Overall, 322 patients were treated with Adriamycin, Bleomycin, Vinblastine, and Dacarbazine regimen, and the Stanford regimen was administered to 31 cases. Most of the patients (57.59\%) had clinically cervical-involved lymph nodes. Furthermore, 31 and 75 patients had axillary and inguinal involvement, respectively. Moreover, 75 cases had involvement in another part of the body, and other patients had no complete medical records. Based on the classical variety, 164 (42.15\%), 94 (24.17\%), and 21 (5.5\%) patients had nodular sclerosis, mixed cellularity, and lymphocyte-rich, respectively.

The estimated results of the two events (relapse and death) using both joint and independent frailty models are presented in Tables 2 and 3 .

Table 2 shows the independent frailty model results. According to this model, by considering individual characteristics, it was concluded that older age (over 55 years) was an effective and important factor in all transitions and endpoints. Moreover, the patients with cervical nodes were more at risk of relapse, compared to those whose other parts of the body were affected (HR01: 0.72 [0.20, 0.88]). Furthermore, a biopsy had also effects on the relapse. The risk of death in patients with hemoglobin less than 10.5 was more than that in others (HR02: 0.28 $[0.14,0.55])$. Among the prognostic factors, gender was well known in Hodgkin disease. Males were more prone to relapse and death, compared to females. In other words, a high mortality rate was noted among males $\left(\mathrm{HR}_{02}: 1.63[1.84,3.18]\right)$. In this model, the variance of frailty was significantly different from zero for transitions 01 and 02 to insignificant for transition 12. This result was obtained using a

\begin{tabular}{|c|c|c|c|c|}
\hline \multicolumn{2}{|c|}{ Three independent frailties for transitions $01,12,02$} & Diagnose $\rightarrow$ Relapse & Relapse $\rightarrow$ Death & Diagnose $\rightarrow$ Death \\
\hline \multirow{5}{*}{ Age } & & $\operatorname{Exp} \beta($ CI 95\%) & $\operatorname{Exp} \beta(\mathrm{CI} 95 \%)$ & $\operatorname{Exp} \beta($ CI 95\%) \\
\hline & $15-34$ & $1.34(0.59-3.06)$ & $1.40(0.302-6.53)$ & $3.21(1.13-4.08)$ \\
\hline & $34-55$ & $2.61(0.94-3.29)$ & $2.93(0.61-13.98)$ & $3.08(0.91-5.49)$ \\
\hline & $>55$ & $3.74(1.21-4.55)$ & $1.96(1.79-44.71)$ & $2.44(1.87-3.10)$ \\
\hline & $<15$ & 1 & 1 & 1 \\
\hline \multirow{2}{*}{ Gender } & Male & $1.28(0.69-2.36)$ & $1.19(0.50-2.86)$ & $1.63(1.84-3.18)$ \\
\hline & Female & 1 & 1 & 1 \\
\hline \multirow{4}{*}{ Location lymph } & Axillary nodes & $0.42(0.76-1.88)$ & $1.61(0.608-4.28)$ & $0.83(0.37-1.87)$ \\
\hline & Inguinal nodes & $0.45(0.28-1.98)$ & $0.99(0.27-3.64)$ & $0.50(0.16-1.57)$ \\
\hline & Cervical nodes & $0.72(0.20-0.88)$ & $1.08(0.104-11.13)$ & $0.51(0.13-2.08)$ \\
\hline & Other & 1 & 1 & 1 \\
\hline \multirow{2}{*}{ Biopsy } & Positive & $2.38(1.04-3.01)$ & $2.47(0.018-5.86)$ & $1.69(0.81-3.44)$ \\
\hline & Negative & 1 & & 1 \\
\hline \multirow{2}{*}{ Aspiration } & Yes & $0.69(0.28-1.66)$ & $2.75(0.015-8.76)$ & $0.63(0.31-1.28)$ \\
\hline & No & 1 & 1 & 1 \\
\hline \multirow{2}{*}{ Hemoglobin } & $>10.5$ & $0.83(0.46-1.49)$ & $0.59(0.264-1.33)$ & $0.28(0.14-0.55)$ \\
\hline & $<10.5$ & 1 & 1 & 1 \\
\hline \multirow{3}{*}{ Classical Hodgkin } & Mixed ellularity & $0.72(0.38-1.36)$ & $1.09(0.461-2.58)$ & $1.04(0.52-2.07)$ \\
\hline & Lymphocyte rich & $0.49(0.20-1.17)$ & $0.77(0.237-2.53)$ & $1.03(0.45-2.35)$ \\
\hline & Nodular clerosis & 1 & 1 & 1 \\
\hline \multicolumn{2}{|c|}{ Variance of the frailty(SE) } & $2.75(1.46)^{*}$ & $1.12(0.815)$ & $0.156(0.0721)^{*}$ \\
\hline \multicolumn{2}{|c|}{ Likelihood cross validation (LCV) } & 2.813 & 2.564 & 3.24 \\
\hline
\end{tabular}




\begin{tabular}{|c|c|c|c|}
\hline \multicolumn{2}{|c|}{ A joint subject-specific frailty term for transitions 01,12} & Diagnose $\rightarrow$ Relapse & Relapse $\rightarrow$ Death \\
\hline \multirow{4}{*}{ Age } & $15-34$ & $1.49(0.76-2.92)$ & $1.22(0.86-2.45)$ \\
\hline & $34-55$ & $2.25(1.07-4.73)$ & $1.62(1.03-2.54)$ \\
\hline & $>55$ & $5.95(2.63-6.43)$ & $2.94(1.53-2.86)$ \\
\hline & $<15$ & 1 & 1 \\
\hline \multirow{2}{*}{ Gender } & Male & $1.17(0.71-1.94)$ & $2.27(1.76-3.21)$ \\
\hline & Female & 1 & 1 \\
\hline \multirow{4}{*}{ Location lymph } & Axillary nodes & $0.44(0.25-0.78)$ & $0.84(0.65-1.07)$ \\
\hline & Inguinal nodes & $0.69(0.33-1.46)$ & $1.76(0.95-2.56)$ \\
\hline & Cervical nodes & $0.46(0.14-1.53)$ & $2.50(1.95-5.86)$ \\
\hline & Other & 1 & 1 \\
\hline \multirow{2}{*}{ Biopsy } & Positive & $3.12(1.63-4.88)$ & $1.73(1.27-4.23)$ \\
\hline & Negative & 1 & 1 \\
\hline \multirow{2}{*}{ Aspiration } & Yes & $1.75(0.97-3.12)$ & $1.61(0.61-2.98)$ \\
\hline & No & & 1 \\
\hline \multirow{2}{*}{ Hemoglobin } & $>10.5$ & $0.66(0.41-1.05)$ & $2.01(1.38-5.67)$ \\
\hline & $<10.5$ & 1 & 1 \\
\hline \multirow{3}{*}{ Classical Hodgkin } & Nodular clerosis & $0.67(0.40-1.11)$ & $1.17(0.89-2.89)$ \\
\hline & Mixed ellularity & $0.47(0.24-0.92)$ & $1.88(1.69-4.67)$ \\
\hline & Lymphocyte rich & 1 & $2.65(1.14-5.01)$ \\
\hline \multicolumn{2}{|c|}{ Variance of the frailty (SE) } & \multicolumn{2}{|c|}{$1.148(0.108)$} \\
\hline \multicolumn{2}{|c|}{ Power coefficient $\zeta$} & \multicolumn{2}{|c|}{$7.143(1.141)$} \\
\hline \multicolumn{2}{|c|}{ Likelihood cross-validation } & \multicolumn{2}{|c|}{2.104} \\
\hline
\end{tabular}

modified Wald test $(1.12 / 0.815<1.64)$. This showed that individual homogeneity was an effective agent in relapse and death. However, the occurrence of death among patients with relapse experience was due to their condition.

According to joint frailty results (Table 3), there was an association between the transition time of relapse (01) and the following death (12). Lack of attention to this correlation may cause underestimating the coefficients and created biases. In this study, the variance of frailty was significantly different from zero: $\alpha=1.148$ (SE [H]: 0.108). Furthermore, the power coefficient was significantly different from zero (W=7.14/1.14). This showed that the incidence of relapse was positively associated with death. Older age, type of lymphocyte, lymphocyte-rich Hodgkin, cervical nodes, and biopsy significantly had an effect on relapse and death in this model.

The conclusions drawn from the previous two models by comparing standard error showed that estimation in the joint frailty model was more accurate and performed better than the independent frailty model. Moreover, the Likelihood CrossValidation (LCV) criterion was proposed to choose the joint frailty model as a better fitting model. The joint frailty model was selected as a lower value of LCV that demonstrated a better model in this regard.

There were also attempts in this study to predict death from joint and shared frailty models, followed by the assessment of its performance. Figure 2 compares the risk of death for both models with the same individual characteristics, including male gender, low hemoglobin, and old age. This prediction was performed according to the existing information, as well as basic and important factors.

The risk of death would be different considering the number of previous recurrences. The occurrence of death will be earlier for patients with at least $\mathrm{j}$ recurrence, compared to patients with exactly $\mathrm{j}$
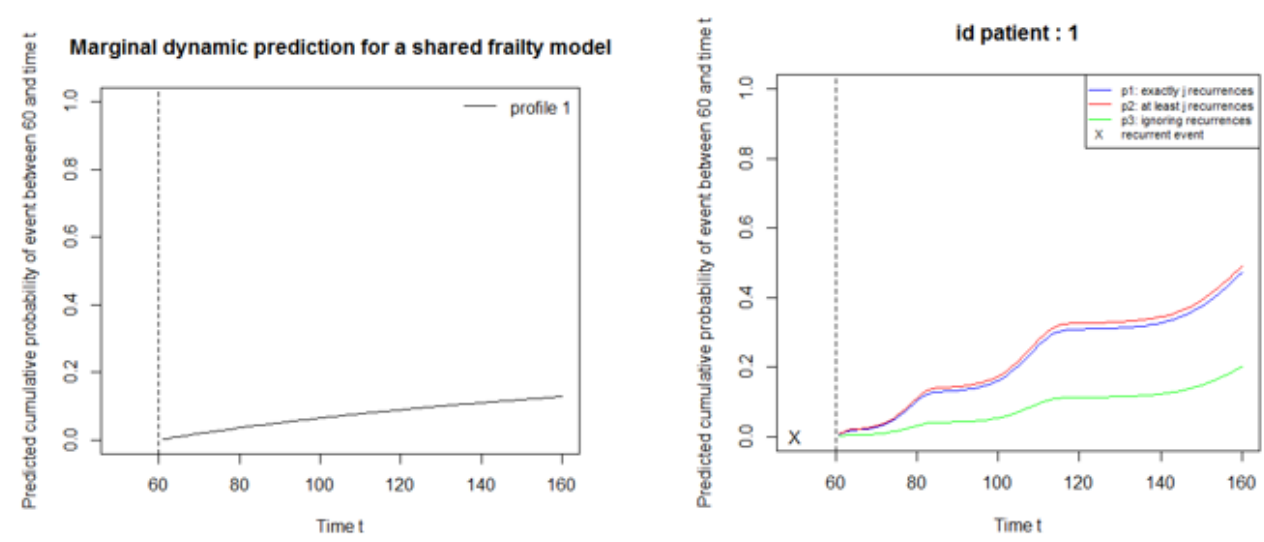

Figure 2. Evolution of the three prediction settings for shared and joint frailty models sharing same characteristics, except for the occurrence of recurrences 
recurrence. However, patients without relapse were more prone to death. In general, each relapse was followed by a new line of treatment that was selected according to the patients' characteristics. Patients with relapse would be treated by a recent and modern treatment approach. This might prevent them from experiencing an unfavorable outcome in comparison to ignoring a relapse of the disease. This is the main reason for focusing on those intermediate events in research. Prediction in the shared frailty model was exactly similar to prediction without any relapse in the joint frailty model.

\section{Discussion}

The most common and popular regression model for survival analysis was the Cox proportional hazards model. However, this approach was unable to offer a wide insight into the duration of the disease and several endpoints. The MSM provided complex survival models for the progression of the disease (8). Numerous studies especially in chronic diseases, such as cancers, utilized the MSM since it brought some new information about the progress of the disease in patients (9-13). As previous literature about HL used the usual method for survival analyses, several states of the disease were evaluated by MSM in this study. This model allowed us to understand the effect of prognostic factors on each state. The addition of the frailty to the MSM, despite the challenge, provided strong survival models to explain some point of heterogeneity among subjects, groups, and different centers (14). In this study, the accommodation of individual heterogeneity to our illness-death model could catch and control unobserved heterogeneity. On the other hand, the association between transition intensities was described in the joint frailty MSM framework. This model reduced the estimator's bias and provided precise predictions. In a study conducted by Mauguen et al., relapse was considered to have a great impact on predicting the death risk. They also took into account that relapse information decreased the prediction error (15). Similar to our results, Mazroui showed that joint modeling made suitable predictions (16). Consistent with the results obtained from this study, they inferred that the best imputation method was the prediction from a joint model. The satisfactory results were in terms of the reduction of standard errors of estimation $(17,18)$. Regarding the clinical aspect of MSM, frailty and joint frailty could help clinicians to avoid the potential reasons for relapse or recommend the most effective treatment.

The evaluation of the prognostic factors provided more precise insight into the disease process, especially in cancer research. Much fundamental decision for optimal treatment depended on accurate knowledge about the prognostic index, and it was very important to have an acute understanding of the diagnosis for remission (19). According to our results, factors, such as old age, male gender, and low level of hemoglobin significantly increased the risk of relapse or death. Moreover, the individual characteristic affected the risk of death or relapse. Numerous studies confirmed the effect of old age on physical functioning and its adverse effect on treatment (20-24). It meant that the ages over 45 or 50 years were diagnosed as a prognostic factor for HL (22). In fact, overall survival and freedom from progression (FFP) depended on the patient's age. It was reported that severe drug-related toxicity was more frequent in older patients, compared to the younger cases $(25,26)$. Hasenclever indicated that the survival rate decreased from $42 \%$ to $5 \%$ in five years for patients up to 34 years who relapsed, compared to those who were between 55 and 56 years. Similar to our results, several studies found age to be a significant and an adverse risk factor since a biologically more aggressive disease presents more comorbidities and more treatment toxicity at an old age (27-31).

Among the prognostic factors, gender was well known in Hodgkin disease. Our results clearly showed male gender was more at risk for death or relapse. These findings were consistent with the results of previously conducted studies $(1,32,33)$. A systematic review considered various epidemiological patterns according to the geographical location. A significant association was reported between male gender and HL disease in Hispanics. In contrast to our result, up to $13.7 \%$ of incidents occurred among females annually (34). Moreover, another controversial result was reported by Jeffery (35), who found some factors (e.g., gender) that were predictive of disease-free survival, relapse, and overall survival. In this retrospective study, out of 163 patients with Hodgkin disease, females experienced relapse more than males (26\% vs. $39 \%$ ). Disease-free survival was significantly different for different genders and complete remission was noted in 78 males and 57 females. However, the overall survival was not influenced by gender. Altogether, females were more fragile, compared to males. Another study showed significant and better outcomes for females, compared to males (37). Consequently, the male gender was identified as an adverse prognostic factor for Hodgkin (37). According to the present study, a lower level of hemoglobin was an unfavorable factor for Hodgkin's disease. This finding was consistent with that of other studies (24, 38-42). In other words, anemia (hemoglobin<10.5) was considered an adverse prognostic factor for overall survival (43). Beirman found that anemia resulted in shorter survival (21). Furthermore, according to the International Prognostic Score, age and lower levels of hemoglobin are the factors that affected FFP (44). In contrast, Paolo found that hemoglobin did not significantly affect survival and freedom from treatment (32). 


\section{Conclusion}

The main objectives of this study were to explore the problem of subject-event dependency in the survival data to provide useful tools to deal with them and propose a solution to take advantage of both the multi-state and frailty methodologies jointly. Although previous studies considered the prognostic factors of Hodgkin's disease, the utilization of the MSM was a relatively novel approach for this cancer. This helped to model different states of the disease, as well as describe the latent factors and the states simultaneously (14). The HL was regarded as a good progress disease in its diagnosis and treatment during years. Therefore, an understanding of the prognostic factors provided a basis for the treatment. It could, in particular, help clinicians avoid the potential reasons for relapse or help them recommend the most effective treatment. Multistate models highlighted the impact of prognostic factors on intermediate events during the disease process. Cancers are among the most dangerous and challenging diseases, in which multistate models are frequently used. Moreover, in such an analysis, patients' prognoses may be predicted over time. In addition, individual characteristics can decrease bias in estimations. Regarding the limitations of this study, one can refer to the small sample size. To estimate the accurate coefficients, a larger sample size is required for each transition and covariate. In the current study, the variables of the stages of the disease were omitted among other variables for a lack of adequate cases in one transition. Overall, despite certain limitations, determination of the prognostic factors can avoid the unfavorable effects of cancer on patients and the quality of their lives.

\section{Acknowledgments}

The valuable contribution of research center of thalassemia and hemoglobinopathy is greatly appreciated. This study was financially supported by the Research Deputy (U-96004) of Jundishapur University of Medical Sciences, Ahvaz, Iran. Furthermore, this study was extracted from an MSc thesis in Biostatistics by Fatemeh Javanmardi.

\section{Footnotes}

Authors' Contribution: ASM and FJ: Conception and design; Analysis and interpretation of the data; Drafting of the article. FR: Conception and design. AA: Drafting of the article. MS: Collection and assembly of data, Drafting of the article. All authors approved the final version of the article for submission.

Conflict of Interests: The authors have no conflict of interest to declare.

Ethical Approval: This study was approved by the Ethics Committee of Ahvaz Jundishapur University of
Medical Sciences, Ahvaz, Iran. (IR.AJUMS.REC. 1396.6).

Funding/Support: This study was financially supported by the Research Deputy (U-96004) of Jundishapur University of Medical Sciences, Ahvaz, Iran.

Financial Disclosure: None.

Informed consent: The written informed consent was obtained from those who willingly agreed to participate in this study.

\section{References}

1. Kuruvilla J, Keating A, Crump M. How I treat relapsed and refractory Hodgkin lymphoma. Blood. 2011;117(16):4208-17. doi: 10.1182/blood-2010-09-288373. [PubMed: 21263152].

2. Johnson P, McKenzie H. How I treat advanced classical Hodgkin lymphoma. Blood. 2015;125(11):1717-23. doi: 10.1182/blood2014-09-551556. [PubMed: 25564404].

3. Zare A, Mahmoodi M, Mohammad K, Zeraati H, Hosseini M, Holakouie-Naieni K. Assessing misspecification of individual homogeneity assumption in multi-state models based on asymptotic theory. J Biostat Epidemiol. 2015;1(3/4):70-9.

4. Lokich JJ, Pinkus GS, Moloney WC. Hodgkin's disease in the elderly. Oncology. 1974;29(6):484-500. doi: 10.1159/000224931. [PubMed: 4140486].

5. Boyle P, Levin B. World cancer report 2008. Geneva: IARC Press, International Agency for Research on Cancer; 2008. P. 15-25.

6. Liquet B, Timsit JF, Rondeau V. Investigating hospital heterogeneity with a multi-state frailty model: application to nosocomial pneumonia disease in intensive care units. $B M C$ Med Res Methodol. 2012;12:79. doi: 10.1186/1471-2288-1279. [PubMed: 22702430].

7. Rondeau V, Mathoulin-Pelissier S, Jacqmin-Gadda H, Brouste V, Soubeyran P. Joint frailty models for recurring events and death using maximum penalized likelihood estimation: application on cancer events. Biostatistics. 2007;8(4):708-21. doi: 10.1093/biostatistics/kxl043. [PubMed: 17267392].

8. Rotolo F, Legrand C. Frailty multi-state models using maximum penalised partial likelihood estimation. Padova, Italy: University of Padova; 2019.

9. Beyersmann J, Wolkewitz M, Allignol A, Grambauer N, Schumacher M. Application of multistate models in hospital epidemiology: advances and challenges. Biom J. 2011;53(2): 332-50. doi: 10.1002/bimj.201000146. [PubMed: 21374697].

10. Meier-Hirmer C, Schumacher M. Multi-state model for studying an intermediate event using time-dependent covariates: application to breast cancer. BMC Med Res Methodol. 2013;13:80. doi: 10.1186/1471-2288-13-80. [PubMed: 23786493].

11. Broët $P$, de la Rochefordière $A$, Scholl $S M$, Fourquet $A$, De Rycke $\mathrm{Y}$, Pouillart $\mathrm{P}$, et al. Analyzing prognostic factors in breast cancer using a multistate model. Breast Cancer Res Treat. 1999;54(1):83-9. doi: 10.1023/a:1006197524405. [PubMed: 10369084].

12. Sutradhar R, Forbes S, Urbach DR, Paszat L, Rabeneck L, Baxter NN. Multistate models for comparing trends in hospitalizations among young adult survivors of colorectal cancer and matched controls. BMC Health Serv Res. 2012;12:353. doi: 10.1186/1472-6963-12-353. [PubMed: 23043307].

13. Conlon AS, Taylor JM, Sargent DJ. Multi-state models for colon cancer recurrence and death with a cured fraction. Stat Med. 2014;33(10):1750-66. doi: 10.1002/sim.6056. [PubMed: 24307330].

14. Rondeau V, Mauguen A, Laurent A, Berr C, Helmer C. Dynamic prediction models for clustered and interval-censored outcomes: investigating the intra-couple correlation in the risk of dementia. Stat Methods Med Res. 2017;26(5):2168-83. doi: 10.1177/0962280215594835. [PubMed: 26184832].

15. Mauguen A, Rachet B, Mathoulin-Pélissier S, MacGrogan G, 
Laurent A, Rondeau V. Dynamic prediction of risk of death using history of cancer recurrences in joint frailty models. Stat Med. 2013;32(30):5366-80. doi: 10.1002/sim.5980. [PubMed: 24030899].

16. Rondeau V, Mazroui Y, Gonzalez JR. Frailtypack: an R package for the analysis of correlated survival data with frailty models using penalized likelihood estimation or parametrical estimation. J Stat Softw. 2012;47(4):1-28.

17. Parast L, Tian L, Cai T. Landmark estimation of survival and treatment effect in a randomized clinical trial. J Am Stat Assoc. 2014;109(505):384-94. doi: 10.1080/01621459.2013.842488. [PubMed: 24659838]

18. Mazroui Y, Mathoulin-Pélissier S, Macgrogan G, Brouste V, Rondeau V. Multivariate frailty models for two types of recurrent events with a dependent terminal event: application to breast cancer data. Biom J. 2013;55(6):866-84. doi: 10.1002/bimj.201200196. [PubMed: 23929494].

19. Putter H, van Houwelingen HC. Frailties in multi-state models: are they identifiable? Do we need them? Stat Methods Med Res. 2015;24(6):675-92. doi: 10.1177/0962280211424665. [PubMed: 22116343].

20. Bierman PJ, Lynch JC, Bociek RG, Whalen VL, Kessinger A, Vose JM, et al. The international prognostic factors project score for advanced Hodgkin's disease is useful for predicting outcome of autologous hematopoietic stem cell transplantation. Ann Oncol. 2002;13(9):1370-7. doi: 10.1093/annonc/mdf228. [PubMed: 12196362].

21. Smolewski P, Robak T, Krykowski E, Blasiñska-Morawiec M, Niewiadomska H, Pluzanska A, et al. Prognostic factors in Hodgkin's disease: multivariate analysis of 327 patients from a single institution. Clin Cancer Res. 2000;6(3):1150-60. [PubMed: 10741746].

22. Bierman PJ, Bagin RG, Jagannath S, Vose JM, Spitzer G, Kessinger A, et al. High dose chemotherapy followed by autologous hematopoietic rescue in Hodgkin's disease: long-term follow-up in 128 patients. Ann Oncol. 1993;4(9):767-73. doi: 10.1093/oxfordjournals.annonc.a058662. [PubMed: 8280658].

23. Fermé $C$, Bastion $Y$, Brice $P$, Lederlin $P$, Diviné $M$, Gabarre J, et al. Prognosis of patients with advanced Hodgkin's disease: evaluation of four prognostic models using 344 patients included in the Group d'Etudes des Lymphomes de l'Adulte Study. Cancer. 1997;80(6):1124-33. [PubMed: 9305714].

24. Kılıçkap S, Barıșta I, Ulger S, Celik I, Selek U, Yıldız F, et al. Clinical features and prognostic factors of Hodgkin's lymphoma: a single center experience. Balkan Med J. 2013;30(2):178-85. doi: 10.5152/balkanmedj.2012.110. [PubMed: 25207097].

25. Balducci L, Phillips DM, Davis KM, Files JC, Khansur T, Hardy CL. Systemic treatment of cancer in the elderly. Arch Gerontol Geriatr. 1988;7(2):119-50. doi: 10.1016/0167-4943(88)90026x. [PubMed: 3046534]

26. Peterson BA, Pajak TF, Cooper MR, Nissen NI, Glidewell OJ, Holland JF, et al. Effect of age on therapeutic response and survival in advanced Hodgkin's disease. Cancer Treat Rep. 1982;66(4):889-98. [PubMed: 7042088].

27. Austin-Seymour MM, Hoppe RT, Cox RS, Rosenberg SA, Kaplan HS. Hodgkin's disease in patients over sixty years old. Ann Intern Med. 1984;100(1):13-8. doi: 10.7326/0003-4819-1001-13. [PubMed: 6691638].

28. Specht L, Nissen NI. Hodgkin's disease and age. Eur J Haematol. 1989;43(2):127-35. [PubMed: 2792320].

29. Davis S, Dahlberg S, Myers MH, Chen A, Steinhorn SC. Hodgkin's disease in the United States: a comparison of patient characteristics and survival in the Centralized Cancer Patient Data System and the Surveillance, Epidemiology, and End Results Program. J Natl Cancer Inst. 1987;78(3):471-8. [PubMed: 3469461].

30. Sutcliffe SB, Wrigley PF, Peto J, Lister TA, Stansfeld AG, Whitehouse JM, et al. MVPP chemotherapy regimen for advanced Hodgkin's disease. Br Med J. 1978;1(6114):679-83. doi: 10.1136/bmj.1.6114.679. [PubMed: 630293].

31. Vaughan Hudson B, MacLennan KA, Easterling MJ, Jelliffe AM, Haybittle JL, Vaughan Hudson G. The prognostic significance of age in Hodgkin's disease: examination of 1500 patients (BNLI report no. 23). Clin Radiol. 1983;34(5):503-6. doi: 10.1016/s0009-9260(83)80150-0. [PubMed: 6617081].

32. Gobbi PG, Comelli M, Grignani GE, Pieresca C, Bertoloni D, Ascari E. Estimate of expected survival at diagnosis in Hodgkin's disease: a means of weighting prognostic factors and a tool for treatment choice and clinical research. A report from the International Database on Hodgkin's Disease (IDHD). Haematologica. 1994;79(3):241-55. [PubMed: 7926973].

33. Haybittle JL, Hayhoe FG, Easterling MJ, Jelliffe AM, Bennett MH, Vaughan Hudson G, et al. Review of British National Lymphoma Investigation studies of Hodgkin's disease and development of prognostic index. Lancet. 1985;1(8435):967-72. doi: 10.1016/s0140-6736(85)91736-2. [PubMed: 2859421].

34. Massini G, Siemer D, Hohaus S. EBV in Hodgkin lymphoma. Mediterr J Hematol Infect Dis. 2009;1(2):e2009013. doi: 10.4084/MJHID.2009.013. [PubMed: 21416003].

35. Jeffery GM, Colls BM, Robinson BA, Fitzharris BM, Atkinson CH. A risk factor for relapse in Hodgkin's disease: female gender? Hematol Oncol. 1989;7(5):345-53. doi: 10.1002/hon.2900070503. [PubMed: 2767620].

36. Klimm B, Reineke T, Haverkamp H, Behringer K, Eich HT, Josting A, et al. Role of hematotoxicity and sex in patients with Hodgkin's lymphoma: an analysis from the German Hodgkin Study Group. J Clin Oncol. 2005;23(31):8003-11. doi: 10.1200/JCO.2005.205.60. [PubMed: 16204002].

37. Gobbi PG, Zinzani PL, Broglia C, Comelli M, Magagnoli M Federico M, et al. Comparison of prognostic models in patients with advanced Hodgkin disease. Promising results from integration of the best three systems. Cancer. 2001;91(8): 1467-78. doi: 10.1002/1097-0142(20010415)91:8<1467::aidcncr1154>3.0.co;2-a. [PubMed: 11301394].

38. Connors JM. Risk assessment in the management of newly diagnosed classical Hodgkin lymphoma. Blood. 2015;125(11): 1693-702. doi: 10.1182/blood-2014-07-537480. [PubMed: 25575542].

39. Löffler M, Dixon DO, Swindell R. Prognostic factors of stage III and IV Hodgkin's disease. Treatment strategy in Hodgkin's disease. Montrouge: John Libbey Eurotext; 1990. P. 89-104.

40. Proctor SJ, Taylor P, Donnan P, Boys R, Lennard A, Prescott RJ. A numerical prognostic index for clinical use in identification of poor-risk patients with Hodgkin's disease at diagnosis. Scotland and Newcastle Lymphoma Group (SNLG) Therapy Working Party. Eur J Cancer. 1991;27(5):624-9. doi: 10.1016/0277-5379(91)90231-2. [PubMed: 1828974].

41. Hasenclever D, Schmitz N, Diehl V. Is there a rationale for highdose chemotherapy as first line treatment of advanced Hodgkin's disease? German Hodgkin's Lymphoma Study Group (GHSG). Leuk Lymphoma. 1995;15(Suppl 1):47-9. doi: 10.3109/10428199509052706. [PubMed: 7767261].

42. MacLennan KA, Vaughan Hudson B, Easterling MJ, Jelliffe AM, Vaughan Hudson G, Haybittle JL. The presentation haemoglobin level in 1103 patients with Hodgkin's disease (BNLI report no. 21). Clin Radiol. 1983;34(5):491-5. doi: 10.1016/s0009-9260(83)80146-9. [PubMed: 6617079].

43. Cuccaro A, Bartolomei F, Cupelli E, Galli E, Giachelia M, Hohaus S. Prognostic factors in hodgkin lymphoma. Mediterr J Hematol Infect Dis. 2014;6(1):e2014053. doi: 10.4084/MJHID.2014.053. [PubMed: 25045461].

44. Moccia AA, Donaldson J, Chhanabhai M, Hoskins PJ, Klasa RJ Savage KJ, et al. International prognostic score in advanced-stage Hodgkin's lymphoma: altered utility in the modern era. Clin Oncol. 2012;30(27):3383-8. doi: 10.1200/JC0.2011.41.0910. [PubMed: 22869887]. 